\title{
Luxaciones puras de codo en pacientes pediátricos: tratamiento conservador y complicaciones asociadas a una patología poco prevalente. Serie de 4 casos Isolated elbow dislocation in pediatric patients: non-operative treatment and
complications associated with an infrequent pathology. Series of 4 cases
}

Dra. Catalina Larrague ${ }^{a}$ Dr. Diego Campelo ${ }^{a}$ Dr. Fernando Díaz Dilernia ${ }^{a}$, Dr. Santiago Bosio ${ }^{a}$ Dr. Rubén Maenza y Dr. Miguel Puigdevall ${ }^{a}$

\section{RESUMEN}

Si bien el codo es la articulación más frecuentemente luxada en niños, representa el 3-6\% de las lesiones en ese sitio. Las luxaciones $\sin$ fracturas asociadas son muy raras y son producto de una caída con el codo en extensión. El paciente consulta por dolor, impotencia funcional y deformidad evidente. La finalidad del tratamiento es restaurar la congruencia articular, lograr estabilidad y minimizar los riesgos de posibles lesiones neurovasculares.

Se presentan 4 pacientes tratados con manejo conservador con excelentes resultados funcionales, incluso aquel que presentó una neuropraxia del mediano con restitución ad integrum.

Según nuestra experiencia, suelen ser lesiones con buena evolución. Se destaca la importancia de un rápido y preciso examen neurovascular, optando, de ser posible, por una conducta expectante ante las lesiones nerviosas. Se resalta la indicación de una inmovilización acotada con movilización temprana que evite rigidez del codo.

Palabras clave: luxaciones articulares, codo, niño, rigidez muscular, paresia.

\section{ABSTRACT}

Even though the elbow is the most often dislocated joint in children, this injury accounts for 3-6 \% of elbow pathology. Dislocations without associated fractures are extremely rare. They result from a fall onto an outstretched hand. The patient is always referred with a painful joint, movement impairment and even clinical deformity. Acute treatment aims to achieve quick reduction and adequate joint stability, avoiding neurovascular injuries.

We sought to analyze the functional outcomes and the complications after non-operative treatment. Our 4 patients

a. Hospital Italiano de Buenos Aires, Instituto de Ortopedia y Traumatología "Carlos E. Ottolenghi". Buenos Aires, Argentina.

\section{Correspondencia:}

Dra. Catalina Larrague:

catalina.larrague@hospitalitaliano.org.ar

Financiamiento: Ninguno.

Conflicto de intereses: Ninguno que declarar.

Recibido: 30-3-2020

Aceptado: 23-10-2020 had excellent functional results at the latest follow-up, and one of them suffered from a median nerve palsy without further consequences.

In our experience, these injuries presented excellent outcomes and we would like to highlight the importance of a quick and precise neurovascular examination with the possibility of non-surgical management of nerve injuries. A short period of immobilization with early rehabilitation should be indicated to avoid joint stiffness.

Key words: joint dislocations, elbow, child, muscle rigidity, paresis.

http: / / dx.doi.org/10.5546/aap.2021.e133

Cómo citar: Larrague C, Campelo D, Díaz Dilernia F, Bosio S, et al. Luxaciones puras de codo en pacientes pediátricos: tratamiento conservador y complicaciones asociadas a una patología poco prevalente. Serie de 4 casos. Arch Argent Pediatr 2021;119(2):e133-e137.

\section{INTRODUCCIÓN}

El codo es la articulación grande luxada con más frecuencia en los niños menores de 10 años. ${ }^{1}$ No obstante, las luxaciones representan el 3-6 \% de todas las lesiones del codo y son significativamente menos habituales que su fractura, ${ }^{2,3}$ lo que hace que las luxaciones puras, sin fracturas asociadas, sean muy raras.

Por lo general, son consecuencia de una caída sobre la mano con el codo en extensión, y, al momento del examen físico, se observa dolor, impotencia funcional y una deformidad evidente. En la urgencia, es importante descartar fracturas asociadas y posibles lesiones neurovasculares, y finalizar, idealmente, con la reducción temprana de la articulación.

Debido a su baja frecuencia en los niños, pocos han sido los casos reportados en la literatura. En general, los reportes publicados describen casos de luxaciones con fracturas asociadas, que suelen evolucionar con una morbilidad aumentada. ${ }^{4} \mathrm{De}$ hecho, tampoco se han encontrado publicaciones en las que se describa el tratamiento conservador de las lesiones del nervio mediano (NM), como se ha registrado en uno de nuestros pacientes. 
El objetivo de esta serie de casos fue analizar los resultados funcionales y las complicaciones de las luxaciones puras de codo en los niños tratadas de forma conservadora.

\section{CASOS CLÍNICOS}

El proyecto fue aprobado por el Comité de Ética de Protocolos de Investigación del Hospital Italiano de Buenos Aires, y los pacientes incluidos firmaron un consentimiento informado que autorizaba la publicación del material de la historia clínica. Se analizaron los resultados funcionales de 4 pacientes menores de 18 años con diagnóstico de luxaciones puras de codo, secundarias a un traumatismo agudo, tratados de forma conservadora entre enero de 2015 y abril de 2019 (Tabla 1).
Los pacientes presentaron un promedio de 7 años de edad. Ninguno refería comorbilidades de relevancia clínica. Fueron atendidos en la Central de Emergencias Médicas Pediátricas del Hospital Italiano de Buenos Aires tras una caída de propia altura sobre sus manos con el codo en extensión.

Clínicamente, se presentaron con dolor, edema e impotencia funcional en el codo. En el inicio, se descartaron lesiones neurovasculares agudas, y luego se realizaron radiografías anteroposterior y de perfil bilaterales para confirmar el diagnóstico y clasificar el desplazamiento, que fue posterolateral en todos los casos. En el mismo día del ingreso, se realizó la reducción cerrada en quirófano, bajo anestesia general, utilizando maniobras de tracción y contratracción.

TABLA 1. Datos demográficos de la serie

\begin{tabular}{lcccc}
\hline Paciente & $\mathbf{1}$ & $\mathbf{2}$ & $\mathbf{3}$ & $\mathbf{4}$ \\
\hline Sexo & Masculino & Masculino & Femenino & Femenino \\
Edad & 7 & 9 & 6 & 7 \\
Lado afectado & Derecho & Izquierdo & Derecho & Izquierdo \\
Desplazamiento & Posterolateral & Posterolateral & Posterolateral & Posterolateral \\
\hline
\end{tabular}

FIgURA 1. Secuencia de imágenes radiológicas

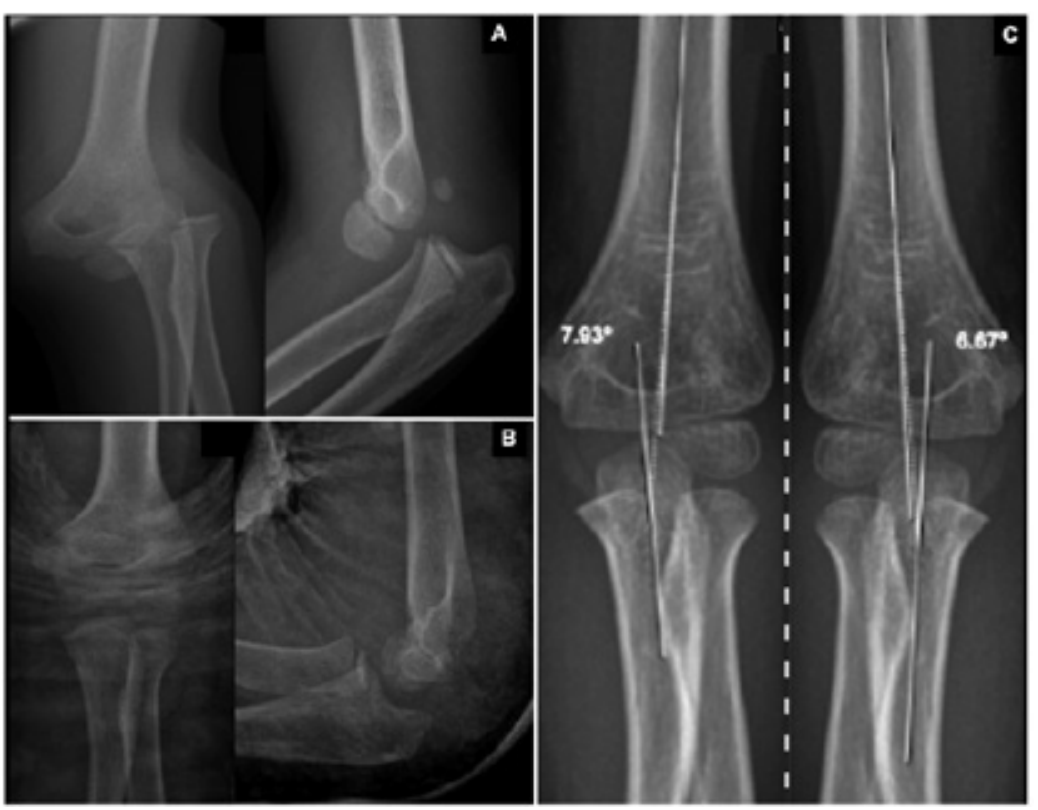

Caso 1. Radiografías de codo izquierdo anteroposterior y de perfil al ingresar. Se observa una luxación posterolateral de codo sin fracturas concomitantes asociadas (A). Radiografías de codo izquierdo anteroposterior y de perfil luego de la exitosa reducción cerrada bajo anestesia con la congruencia articular correspondiente (B). Radiografías anteroposteriores de codo derecho e izquierdo posreducción que comparan la medición del ángulo de carga en ambos codos (C). 
Bajo radioscopía intraoperatoria, se constató la congruencia articular adecuada y se evaluó la estabilidad de la articulación mediante maniobras de estrés. En todos los pacientes, se logró la reducción cerrada satisfactoria, y se presentó una estabilidad adecuada frente al estrés en varo-valgo (Figura 1). Se repitió el examen neurovascular y luego se efectuó la inmovilización del codo con un yeso braquiopalmar a $90^{\circ}$.

El seguimiento promedio de la serie fue de 38 meses. En los controles ambulatorios, fueron evaluados con radiografías y tomografía para constatar la congruencia articular y descartar lesiones concomitantes. En la incidencia anteroposterior, se midió el ángulo de carga y se lo comparó con el codo contralateral, tomándose como valor normal una desviación en valgo de $5-15^{\circ}$. La evaluación funcional constató la estabilidad del codo, mediante maniobras de estrés, y la funcionalidad articular, utilizando el puntaje de Mayo (Mayo Elbow Performance Score; MEPS, por sus siglas en inglés) y el puntaje Disabilities of the Arm, Shoulder and Hand (DASH). ${ }^{5}$

Todos los pacientes tuvieron resultados funcionales excelentes y ninguno presentó nuevos episodios de inestabilidad, cambios en el ángulo de carga comparado con el codo contralateral ni lesiones vasculares asociadas al final del seguimiento (Tabla 2). En cuanto a las lesiones nerviosas, uno de los pacientes sufrió una neuropraxia del NM posterior a la reducción cerrada. Presentó dolor intenso de tipo neuropático en el territorio de inervación, dificultad para realizar la pinza superior, hiperalgesia y disminución de la sensibilidad protopática y vibratoria en los tres primeros dedos de la mano derecha. En imágenes de resonancia magnética nuclear (RMN) del codo, se detectaron hallazgos compatibles con lesión del nervio (Figura 2). El paciente fue inmovilizado con un yeso braquiopalmar por 16 días y luego fue tratado con terapia ocupacional, que logró la restitución ad integrum tras 6 meses de rehabilitación. Otro paciente de la serie presentó limitación a la extensión del codo luego de 21 días de inmovilización. Se le indicó la rehabilitación kinesiológica durante 1 mes, y se alcanzó la extensión completa y una puntuación de Mayo de 100 puntos.

\section{DISCUSIÓN}

Las luxaciones de codo representan el 3-6\% de todas las lesiones de codo en la población general; no obstante, es raro encontrarlas en los niños sin fracturas asociadas. ${ }^{1,2}$ Debido a la inmadurez esquelética, el tejido óseo es más débil que el ligamentario, y, en consecuencia, un mayor estrés aplicado sobre el codo, habitualmente, resulta en una lesión ósea y no en una luxación aislada., Tienen su pico de incidencia en la segunda década de la vida debido al cierre parcial del cartílago de crecimiento de los huesos que conforman el codo. $^{2}$ El lado no dominante ${ }^{1}$ es el más afectado y el mecanismo descrito con mayor frecuencia es la caída sobre la mano con el codo en extensión.

Las luxaciones de codo se clasifican en posteriores, anteriores y divergentes según el desplazamiento del cúbito con respecto al húmero. ${ }^{4}$ Cuando el desplazamiento es posterior, se pueden subclasificar en posterolaterales y posteromediales. El $90 \%$ de las luxaciones de codo son posteriores, y el $70 \%$ de las mismas son posterolaterales. ${ }^{6}$

El tratamiento de elección es la reducción precoz, en la mayoría de los casos, realizando una tracción simple en el eje. ${ }^{7}$ Múltiples autores evidenciaron la relación directa entre el tiempo de inmovilización, la incidencia de rigidez y la limitación a la extensión..$^{7-10}$ Robinson y col., sugirieron una corta inmovilización de 3 a 10 días luego de la reducción y continuar con una rehabilitación temprana. Esto se debe a que la secuela más frecuente es la pérdida de la extensión, lo que se asocia directamente con

TABLA 2. Tratamiento, evaluación posoperatoria y complicaciones

\begin{tabular}{lcccc}
\hline Paciente & $\mathbf{1}$ & $\mathbf{2}$ & $\mathbf{3}$ & $\mathbf{4}$ \\
\hline Días de inmovilización & 16 & 21 & 8 & 19 \\
Estabilidad & Sí & Sí & Sí & Sí \\
Ángulo de carga & $6,67^{\circ}$ & $11,03^{\circ}$ & $12,34^{\circ}$ & $7,85^{\circ}$ \\
Complicaciones & Neuropraxia del mediano & Rigidez & - & - \\
Puntaje de Mayo & 100 & 100 & 0 & 100 \\
Puntaje DASH & 0 & 0 & 39 meses & 29 meses \\
Seguimiento & 31 meses & 53 meses &
\end{tabular}

DASH: Disabilities of the Arm, Shoulder and Hand. 
el tiempo de inmovilización prolongado. ${ }^{10}$ Se cree que, luego de la reducción, la estabilidad se mantiene por el contorno de las superficies articulares, lo que permite la movilización y, así, prevenir la rigidez. ${ }^{8}$ En nuestra serie, los pacientes que no presentaron rigidez posterior a la inmovilización fueron aquellos inmovilizados por menos de 17 días.

Las lesiones neurovasculares son raras, y las más frecuentes son las del nervio cubital, con una incidencia del 6,3\% $\%{ }^{11}$ Además, las lesiones del NM resultan aún más infrecuentes y se reportan en un $3 \%$ de los casos. Su compresión genera isquemia $y$, secundariamente, fibrosis del tejido neural. ${ }^{12}$ Se han reportado casos de compresión del NM en el contexto de luxaciones de codo, y todos ellos fueron tratados con su descompresión quirúrgica. ${ }^{12-15}$ En nuestro caso, el paciente presentó una recuperación ad integrum luego de una intensa rehabilitación sin tener que recurrir a una nueva intervención quirúrgica. Por último, se obtuvieron excelentes resultados funcionales al final del seguimiento ( $>90$ en el $M E P S)$ en los cuatro pacientes, incluso en aquel que sufrió la neuropraxia del NM. No hubo alteraciones en la medición del ángulo de carga
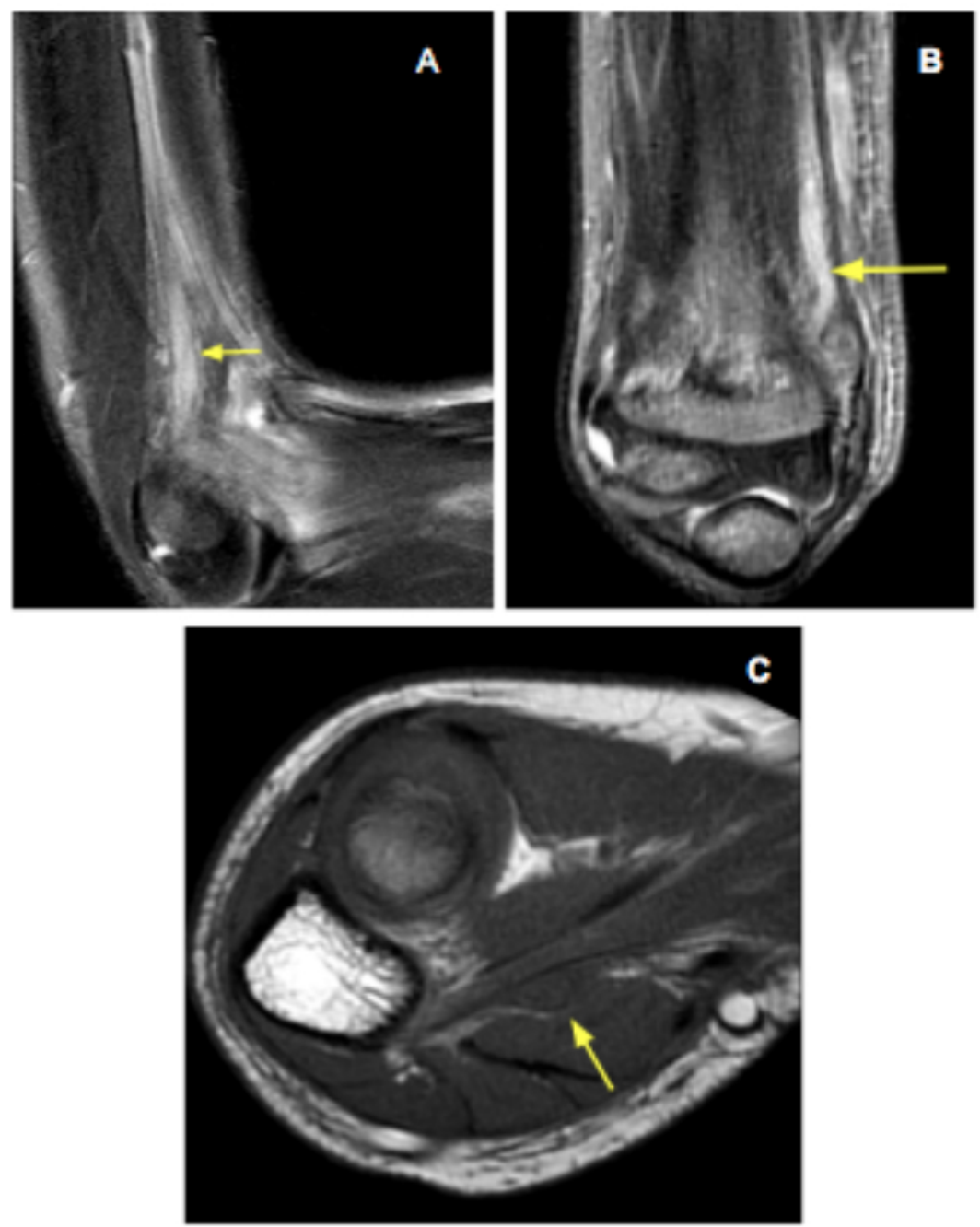

Caso 1. Imágenes de la resonancia magnética nuclear de codo derecho. Corte sagital (A) y corte coronal (B) en secuencia STIR (Short Tau Inversion Recovery). Corte coronal en secuencia T1 (C). Se evidencia el engrosamiento del tejido nervioso y la pérdida de la arquitectura fascicular habitual del nervio, hallazgos compatibles con lesión por distensión del nervio. 
posterior a la reducción, comparado con el codo contralateral.

Las luxaciones puras de codo en los pacientes pediátricos constituyen una patología poco frecuente y, en nuestro Centro, solo se han encontrado 4 casos en 4 años de estudio. A pesar de ser un estudio retrospectivo y con una casuística limitada, las luxaciones puras de codo en los niños parecen ser lesiones benignas y de buena evolución. Es importante que el ortopedista infantil, el médico pediatra y el de familia conozcan la importancia de realizar un examen neurovascular completo para descartar y evitar lesiones asociadas. Asimismo, es fundamental la realización de la reducción cerrada en agudo y considerar el manejo expectante ante la aparición de una lesión nerviosa.

\section{REFERENCIAS}

1. Murphy RF, Vuillermin C, Naqvi M, Miller PE, et al. Early Outcomes of Pediatric Elbow Dislocation-Risk Factors Associated With Morbidity. J Pediatr Orthop. 2017; 37(7):440-6.

2. Kaziz H, Naouar N, Osman W, Ayeche M. Outcomes of paediatric elbow dislocations. Malays Orthop J. 2016; 10(1):44-9.

3. Uhl TL, Gould M, Gieck JH. Rehabilitation after posterolateral dislocation of theelbow in a collegiate football player: a case report. J Athl Train. 2000; 35(1):108-10.

4. Kuhn MA, Ross G. Acute elbow dislocations. Orthop Clin North Am. 2008; 39(2):155-61.

5. Angst F, Schwyzer HK, Aeschlimann A, Simmen BR, et al. Measures of adult shoulder function: Disabilities of the Arm, Shoulder, and Hand Questionnaire (DASH) and its short version (QuickDASH), Shoulder Pain and Disability Index
(SPADI), American Shoulder and Elbow Surgeons (ASES) Society standardized shoulder assessment form, Constant (Murley) Score (CS), Simple Shoulder Test (SST), Oxford Shoulder Score (OSS), Shoulder Disability Questionnaire (SDQ), and Western Ontario Shoulder Instability Index (WOSI). Arthritis Care Res (Hoboken). 2011; 63(Suppl 11):S174-88.

6. LieberJ,ZundelSM, Luithle T, Fuchs J, et al. Acute traumatic posterior elbow dislocation in children. J Pediatr Orthop B. 2012; 21(5):474-81.

7. Robinson PM, Griffiths E, Watts AC. Simple elbow dislocation. Shoulder Elbow. 2017; 9(3):195-204.

8. De Haan J, Schep NWL, Tuinebreijer WE, Patka P, et al. Simple elbow dislocations: a systematic review of the literature. Arch Orthop Trauma Surg. 2010; 130(2):241-9.

9. Di Gennaro GL, Spina M, Fosco M, Antonioli D, et al. Dislocations of the elbow in children: long-term followup. Musculoskelet Surg. 2013; 97(Suppl 1):3-7.

10. Panteli M, Pountos I, Kanakaris NK, Tosounidis TH, et al. Cost analysis and outcomes of simple elbow dislocations. World J Orthop. 2015; 6(7):513-20.

11. O'Callaghan PK, Freeman K, Davis LC, Murphy RF. A rare case of type 2 entrapment of the median nerve after posterior elbow dislocation with MRI and ultrasound correlation. Skeletal Radiol. 2019; 48(10):1629-36.

12. Dubey V, Saify A, Samant A, Shahane S. Median Nerve Entrapment after Elbow Dislocation and the Role of Ultrasonography - A Case Report. J Orthop Case Rep. 2017; 7(3):21-4.

13. Wenger A, Berger J, Piza-Katzer H. Nervenverletzungen nach Ellenbogenluxationsfraktur im Kindesalter. Unfallchirurg. 2016; 119(8):690-7.

14. Petratos DV, Stavropoulos NA, Morakis EA, Matsinos GS. Median nerve entrapment and ulnar nerve palsy following elbow dislocation in a child. J Surg Orthop Adv. 2012; 21(3):157-61.

15. Simon D, Masquijo JJ, Duncan MJ, Kontio K. Intra-articular median nerve incarceration after spontaneous reduction of a pediatric elbow dislocation: case report and review of the literature. J Pediatr Orthop. 2010; 30(2):125-9. 